\title{
TRANSFORMATION KINETICS ON MECHANICAL ALLOYING
}

\author{
Igor F. Vasconcelos* \\ Department of Physics, University of Notre Dame, \\ 225 Nieuwland Science Hall, 46556, Notre Dame, IN, USA \\ Reginaldo S. de Figueiredo \\ LG. Philips Displays - GDE - TZ 1, Zwaanstraat 2, \\ PO Box 80\%, 5600 AV Eindhoven, The Netherlands
}

October 26, 2018

\begin{abstract}
We propose and develop a model as an attempt to describe the local mechanism of mechanical alloying. This model is based on the observation of global parameters, such as the volumes of the various phases present in the material, and their transformations during the process. The model is applied to milled $\mathrm{Fe}-\mathrm{Cu}$ and $\mathrm{Fe}-\mathrm{N}$ experimental results obtained in previous works [1, 2. In the milling of $\mathrm{Fe}-\mathrm{Cu}$ system, four stages, whose features are in accordance with experimental observation, are identified:(i) energy storage; (ii) initiation of reaction and rate increase; (iii) decrease of the reaction rate and (iv) stationary stage. No energy storage stage is observed and no saturation is achieved in the milling of Fe-N system. Moreover, we propose a modification on the global kinetic law introduced in 12 based on the different nature of $\mathrm{Fe}-\mathrm{Cu}$ and $\mathrm{Fe}-\mathrm{N}$ systems. This modification allows the extension of the validity of this law.
\end{abstract}

${ }^{*}$ Corresponding author. E-mail: idevasco@nd.edu 


\section{Introduction}

In classical studies of phase transformations, kinetic laws allow a global description of volume phase as a function of time evolution thus distinguishing them from growth laws. However, growth laws translate a local microscopic modeling of phase transformation phenomena and provide the construction of kinetic laws from microscopic observation and (or) models. Both approaches exist in mechanical alloying (MA) although they are rarely explicit in the literature.

MA is a cheap, efficient, and flexible technique (see the survey by Suryanarayana [5]) which has attracted much attention from scientists of various research areas. Recently, MA has been used to produce materials for biological application [4] and to investigate the spin-glass character of nanograin boundaries [6], amongst many other applications. Although much of the research is based on empirical conclusions obtained directly from experimental results, there has been some attempts to make a quantitative description of MA. A quantitative description is very important and necessary in order to provide a way to establish predictable capabilities for the MA processes [3].

Abdellaoui and Gaffet [7, 8] have shown that neither the shock energy nor the shock frequency govern the $\mathrm{Ni}_{10} \mathrm{Zr}_{7}$ amorphization process in a planetary ball mill if taken into account separately. Only the injected shock power is responsible for the ball milled final product. In another study of $\mathrm{Ni}-\mathrm{Zr}$ amorphization, Chen et al. 9], using a vertical vibrating frame grinder, have found that these compounds become fully amorphized if a defined milling intensity $(I)$ is greater than a threshold value. The milling intensity is defined as:

$$
I=\frac{M_{b} V_{\max } f}{M_{p}},
$$

where $M_{b}$ is the ball mass, $V_{\max }$ is the maximum velocity of the ball, $f$ the impact frequency, and $M_{p}$ the mass of the powder in the vial. Therefore, according to [9], the same final product can be achieved by transfering large momentum with low frequencies or small momentum with high frequencies. These two approaches, while successful in describing individual processes in particular types of mill, have some built in difficulties that prevent them to fully describe a general MA experiment. The shock power proposed in [7, 8] is a function of balls' kinetic energy and impact frequency. However, in a planetary mill, both parameters are functions of the velocity of the balls in the sense that the faster a ball moves, the higher its kinetic energy and the 
more times it will suffer impacts. Moreover, in the vertical vibrating mill used to investigate the role of the milling intensity [9], the shock frequency is determined by the inverse of the time taken by ball to move up and down. This time is proportional (and as a consequence the frequency is inversely proportional) to potential energy. The velocity $V_{\max }$ is itself proportional to potential energy which makes the product $V_{\max } f$ close to a constant value. These two approaches also neglect the importance of the material's response to the impacts.

MA is a "far from equilibrium technique" and despite its dynamic characteristics Yavari et al. [10, 11] have employed equilibrium thermodynamics to explain the MA mechanism. Pure elements form nanocrystalline structures after severe mechanical deformation and the energy stored in the large grain boundaries could be the driving force during alloying. Examining the immiscible $\mathrm{Fe}-\mathrm{Cu}$ system prepared by mechanical alloying, Yavari et al. have shown that the available interfacial energy is not enough to compensate the positive enthalpy of mixing. However, repeated fractures break the particles into very small fragments with high free energies which dissolve themselves into the matrix by the Gibbs-Thomson effect.

Matteazzi et al. [12] have proposed a global kinetic law for MA of iron carbides:

$$
V(t)=k e^{-b t^{c}} .
$$

where $V(t)$ is the transformed phase volume and $k, b$ and $c$ are empirical constants. They have proposed that $c$ is connected to impact energies and $b$ is related to "work conditions". The value of $k$ sets the scale.

In a previous work [1, we reported a discussion about the MA process based on experimental and numerical results. A nanocrystaline fcc$\mathrm{FeCu}$ solid solution was obtained by MA and analyzed by x-ray diffraction, Mössbauer spectroscopy, and differential calorimetry. In addition, simulations of the material behavior during uniaxial deformations, compatible with what we expect on an impact were performed using molecular dynamics. We claimed that the number of balls in the mill vial, which is related to impact frequency, did not affect the energy transfer processes, but rather only the reaction rates. This finding has recently been confirmed by Girot et al. [13]. We also showed that a local description of MA process is necessary in order to understand its driving mechanisms and that many factors such as; powder deformation by the milling tools, chemical and physical properties of materials, strain/stress fields, and collision energies have to be taken in 
account together to describe MA. This view was recently raised in the review by Takacs [14.

In this contribution we propose and develop a model as an attempt to describe the local mechanism of MA. This model is based on the phase volume transformations during the process and is applied to milled $\mathrm{Fe}-\mathrm{Cu}$ and $\mathrm{Fe}-\mathrm{N}$ experimental results obtained previously [1, 2].

\section{The Model}

Our first step to understand MA mechanisms is to investigate how the phase transformations are driven in the course of the process. This can be done using measured global parameters such as the phase volumes. If a given phase $\alpha$ occupies a volume $V_{\alpha}(t)$ in the total volume $V$, then the concentration $X_{\alpha}(t)$ is given by:

$$
X_{\alpha}(t)=\frac{V_{\alpha}(t)}{V} .
$$

MA is essentially a discrete process characterized by discrete energy transfers taking place at individual impacts [15], which means that only a small fraction of the powder is processed at each event. Moreover, a particle of powder under an impact has no memory from the previous impacts [1. Significant powder transformations occurring during milling impacts are confined in a small volume $v$ which is the powder volume trapped by the milling tools, as suggested in 13 . The $\alpha$ phase volume in $v$ is $v_{\alpha}$ and assuming a homogeneous powder [16], the relative concentration is then given by:

$$
X_{\alpha}(t)=\frac{v_{\alpha}(t)}{v}
$$

Let us consider that $\alpha$ phase is created after a MA driven solid state reaction between two other phases $\beta$ and $\gamma$. The volume created or transformed $\Delta v_{\alpha}$ after an impact involving a $\beta \gamma$ interface is a function of the probability to find a $\beta \gamma$ phases reaction $\left(P_{\beta \gamma}(t)\right)$ and tribochemical activity $\left(\xi_{\beta \gamma}^{\alpha}(t)\right)$ given by:

$$
\Delta v_{\alpha}(t) \longrightarrow F\left(P_{\beta \gamma}(t), \xi_{\beta \gamma}^{\alpha}(t)\right)
$$

The word tribochemical includes many aspects such as chemical activities, morphology of components and impact characteristics (energy, frequency, 
etc.). In a first approximation eq. 5 can be written as:

$$
\Delta v_{\alpha}(t)=\sum_{\beta \gamma} P_{\beta \gamma}(t) \xi_{\beta \gamma}^{\alpha}(t) v
$$

This summation is carried out over all $\beta \gamma$ phase reactions which could possibly lead to production of $\alpha$.

A formal description of $\xi_{\beta \gamma}^{\alpha}(t)$ is quite difficult; however, it is possible to describe the volume of a phase during the total milling time $\Delta t$. The evolution of the $\alpha$ phase global volume $\left(\Delta V_{\alpha}(t)\right)$ in the course of time interval $\Delta t$ is given by:

$$
\Delta V_{\alpha}(t)=\Delta v_{\alpha}(t) \times f \Delta t=\sum_{\beta \gamma} P_{\beta \gamma}(t) \xi_{\beta \gamma}^{\alpha}(t) v f \Delta t
$$

where $f$ is the impact frequency and $f \Delta t$ is the number of impacts during the time interval $\Delta t$.

The probability to find a $\beta \gamma$ interface which can react is given by the relative surface areas of the $\beta$ and $\gamma\left(S_{\beta}(t)\right.$ and $\left.S_{\gamma}(t)\right)$ phase grains in the volume $v$ :

$$
P_{\beta \gamma}(t)=\frac{S_{\beta}(t) S_{\gamma}(t)}{S_{T}^{2}},
$$

where $S_{T}$ is the total surface area in the volume $v$. Considering spherical grains, eq. 8 can be written as:

$$
P_{\beta \gamma}(t)=\frac{X_{\beta}(t) X_{\gamma}(t)}{r_{\beta} r_{\gamma}}\left(\sum_{i} \frac{X_{i}(t)}{r_{i}}\right)^{-2}
$$

where $r_{i}$ is the particle radius of phase $i$ and the summation is carried out over all the phases in the volume $v$.

This analysis can be greatly simplified if we consider two-phased systems, composed by phases $\varepsilon$ and $\delta$. The possible reactions between the phases are

$$
\begin{gathered}
\varepsilon+\varepsilon \longrightarrow \Delta v_{\varepsilon}=0, \Delta v_{\delta}=0, \\
\delta+\delta \longrightarrow \Delta v_{\varepsilon}=0, \Delta v_{\delta}=0, \\
\varepsilon+\delta \longrightarrow \Delta v_{\varepsilon}<0, \Delta v_{\delta}>0 \text { or } \Delta v_{\varepsilon}>0, \Delta v_{\delta}<0 .
\end{gathered}
$$

The summation in eq. 7 is reduced to just one term to translate the $\varepsilon$ (or $\delta$ ) volume phase evolution in such a way that:

$$
\Delta V_{\varepsilon}(t)=P_{\varepsilon \delta}(t) \xi_{\varepsilon \delta}^{\varepsilon}(t) v f \Delta t
$$


In this way, the tribochemical activity $\xi_{\varepsilon \delta}^{\varepsilon}(t)$ can be written as:

$$
\xi_{\varepsilon \delta}^{\varepsilon}(t)=\frac{\Delta V_{\varepsilon}(t)}{\Delta t} \frac{1}{P_{\varepsilon \delta}(t) v f} .
$$

Equation 14 presents at the same time a kinetic factor $\left(\frac{\Delta V_{\varepsilon}(t)}{\Delta t}\right)$, a term of chemical nature $\left(P_{\varepsilon \delta}(t)\right)$ and a "condition of milling" term $(v f)$. The probability of a $\varepsilon \delta$ interface is given by eq. 9 and can be written as:

$$
P_{\varepsilon \delta}(t)=\frac{X_{\varepsilon}(t) X_{\delta}(t) r_{\varepsilon} r_{\delta}}{\left[X_{\varepsilon}(t) r_{\delta}+X_{\delta}(t) r_{\varepsilon}\right]^{2}},
$$

and if the particles radii are comparable $\left(r_{\varepsilon} \approx r_{\delta}\right)$, eq. 15] can be reduced to:

$$
P_{\varepsilon \delta}(t)=X_{\varepsilon}(t)\left[1-X_{\varepsilon}(t)\right]
$$

since $X_{\varepsilon}+X_{\delta}=1$. Furthermore, if the impact frequency is high, we get:

$$
\frac{\Delta V_{\varepsilon}(t)}{\Delta t} \approx \frac{d V_{\varepsilon}(t)}{d t}=\frac{d X_{\varepsilon}(t)}{d t} V
$$

Using eq. 16] and 17 with eq. 14 we obtain the proportionality relation:

$$
\xi^{\varepsilon}(t) \propto \frac{d X_{\varepsilon}(t)}{d t} \frac{1}{X_{\varepsilon}(t)\left[1-X_{\varepsilon}(t)\right]} .
$$

At this point we drop the lower indices $\varepsilon$ and $\delta$ because they are no longer necessary. Equation 18 describes the time evolution tribochemical activity to destroy (or create) the $\varepsilon$ phase. The same result applies to $\delta$.

\section{Fe-Cu System}

We used the $\mathrm{Fe}_{55} \mathrm{Cu}_{45}$ system studied in a previous work [1] to perform an analysis of phase volume evolution as described in eq. 7 . We used a Fritsch Pulverisete 5 planetary ball mill to perform two sets of experiments, with 4 and 12 balls in the mill vial. The rotational speed used was $350 \mathrm{rpm}$ for both experiments. The Fe- $\mathrm{Cu}$ samples were prepared by ball milling using commercial $\mathrm{Fe}$ and $\mathrm{Cu}$ metallic powders. In the course of milling, the iron atoms are incorporated into the fcc copper matrix so that at any time during the process, there are fractions of the initial bcc Fe and fcc $\mathrm{Cu}$ and an amount 
of transformed fcc FeCu solid solution. The observed trend is that of total incorporation of iron by the fcc matrix resulting on fcc $\mathrm{FeCu}$ alone [1, 17. Therefore, in the crystallographic point of view, the system consists of a bcc phase $(\mathrm{Fe})$ that decreases gradually and a fcc phase $(\mathrm{Cu}+\mathrm{FeCu})$. We applied the model proposed in this contribution to analyze the bcc phase's kinetics of transformation.

The grain size evolution presents a similar behavior for both bcc and fcc phases and $r_{b c c} \approx r_{f c c}$, (table 1 and fig. 1). Thus, the evolution of tribochemical activity to destroy the bcc phase can be followed by (eq. 18):

$$
\xi^{b c c}(t) \propto \frac{d X_{b c c}(t)}{d t} \frac{1}{X_{b c c}(t)\left[1-X_{b c c}(t)\right]} .
$$

The evolution of phase concentration $X_{b c c}(t)$ can be deduced by the magnetic hyperfine field distributions (BHF) obtained by Mössbauer spectroscopy (fig. 2). Bcc concentration is the area under the spectra with BHF greater than 30 T. Figure 3 shows the evolution curves for the bcc phase volume in the course of milling.

The tribochemical activities versus milling time for the two experiments described are shown in fig. 4. The curves were calculated using eq. 19] and the data in Fig. 3. The scales, however, are not the same because impact frequencies, which are related to the number of balls, are different for each experiment. Figure 5 shows $\xi$ vs. time for the two experiments rescaled by number of balls, in such a way that we get tribochemical activities per ball versus milling time per ball.

\section{Fe-N System}

The $2 \mathrm{Fe}+\mathrm{Fe}_{2} \mathrm{~N} \longrightarrow \mathrm{Fe}_{4} \mathrm{~N}$ reaction obtained by MA studied in a previous work [2] is also a good application for the model discussed in this contribution. The transformation was followed through the volume fraction of $\mathrm{Fe}_{2} \mathrm{~N}$ since the existence domain of this paramagnetic phase is relatively narrow. In addition to this, the system $2 \mathrm{Fe}+\mathrm{Fe}_{2} \mathrm{~N}$ is two-phased, composed by a paramagnetic and a ferromagnetic phase; also the reaction product $\left(\mathrm{Fe}_{4} \mathrm{~N}\right)$ is ferromagnetic. Therefore, the phase volume of the paramagnetic $\mathrm{Fe}_{2} \mathrm{~N}$ can be easily separated by Mössbauer spectroscopy. It is the area of the doublet subspectra of Fig. 6. The $\mathrm{Fe}_{2} \mathrm{~N}$ paramagnetic phase volume evolution is shown in Fig. 7. 
The Fe- $\mathrm{N}$ equilibrium phase diagram is composed by 4 crystallographic phases named $\alpha-F e, \gamma^{\prime}-F e_{4} N, \varepsilon-F e_{2.1-3.6} N$ and $\delta-F e 2 N$. At high temperature one also finds a gamma phase $\gamma-F e_{X} N$ and a epsilon nitrogen poor $\varepsilon-F e_{4} N$. One can also find a metastable long range ordered phase $\alpha^{\prime \prime}-$ $F e_{16} N_{2}$. However, Fe-N phases obtained by milling are quite distinct. Milling of $\gamma^{\prime}-\mathrm{Fe}_{4} \mathrm{~N}$ and $2 \mathrm{Fe}+\mathrm{Fe}_{2} \mathrm{~N}$ leads to a hexagonal phase which presents different nitrogen distribution. Moreover, milling of $4 F e+F e_{4} N$ leads to a hyperferrite instead of the well ordered $\alpha^{\prime \prime}-F e_{16} N_{2}$ [2, 18, 19, 20, 21, 22]. The large domain of epsilon phases, the wide range of the Nitrogen distribution, and the nanocrystalline character of these phases unable any attempt to distinguish nitrogen compositions in epsilon phases. What can be observed is that during most of the milling of $2 \mathrm{Fe}+\mathrm{Fe}_{2} \mathrm{~N}$, the system is mainly composed of $\alpha-F e, \varepsilon-F e_{2.1-3.6} N$ and $\delta-F e_{2} N$. Previous studies about Curie temperature 23] aren't useful because differences in Nitrogen composition lead to diverse magnetic behavior [24]. Indeed, almost any variation on Nitrogen content leads to appearance of ferromagnetic phases, with only $\delta-\mathrm{Fe}_{2} \mathrm{~N}$ remaining paramagnetic.

After just few minutes of alloying it is no longer possible to distinguish between different phases by X-ray diffraction. The local description provided by Mössbauer spectroscopy cannot distinguish Fe atoms without $\mathrm{N}$ as near neighbors from $\alpha-F e$. Even by transmission electron microscopy that separation is no longer possible [24]. Therefore it is only possible to distinguish between ferromagnetic and paramagnetic iron in milled iron nitrides and on the magnetic point of view, the system is composed by two phases: one paramagnetic and one ferromagnetic. Since there are no direct link between tribochemical activity and crystallography (tribochemical activity is a parameter to measure a given activity), this concept can be perfectly applied here.

Either phase is suited for the kind of analysis performed in this paper. Due to experimental convenience, the paramagnetic phase was chosen. The evolution of tribochemical activity to destroy the paramagnetic phase can be followed by eq. 18:

$$
\xi^{P}(t) \propto \frac{d X_{P}(t)}{d t} \frac{1}{X_{P}(t)\left[1-X_{P}(t)\right]} .
$$

The tribochemical activity versus milling time for the Fe-N system studied is showed in fig. 8. The curve was calculated using eq. 20] and data in Fig. [7. 


\section{Discussions}

The two curves in Fig. 4 are not in the same scale because the number of balls, which influences the number of impacts per unit time, is different in each case. We assume the number of impacts per unit time between balls and the vial wall equals the number of balls in the vial. In this way, there are at least $n=3$ times as many impacts in the experiment with 12 balls. A correction to be made refers to the different number of impacts between balls in each experiment. It is virtually impossible to calculate such a number due to the nearly unpredictable dynamics of the balls in the vial. However, we empirically estimate this correction to be $1 / 5$ of $n$. Therefore, there are about $n=3.6$ as many impacts in the 12 balls case. To bring both curves to scale, we modify the 12 balls curve by multiplying time (and dividing $\xi$ ) by $n$. Furthermore, we multiply the time axis (and divide the $\xi$ axis) by 4 to obtain tribochemical activity $v s$. time, per ball. In this way, we get the two curves in Fig. 5 which represent the tribochemical activity time evolution we would obtain if there were only one ball in the vial. The curves in Fig. 5 are very similar indicating that the number of balls (impact frequency) does not play an important role on the final product.

The behavior of the curves in Fig. 5 reveals four distinguished stages. The first one is a static activation stage where the system only stores energy and no chemical reaction occurs [14]. The second stage is characterized by the start of reaction followed by an increase of reaction speed. In fact, the reaction occurs only in the bcc/fcc interface and so this acceleration is expected when total surface increases (grain size decreases) like in fig. 1. The third stage is characterized by a decrease in the reaction rate. During this stage, the amount of iron dissolved in the copper matrix approaches the solubility limit of $\approx 60 \%$ [25, 26, which explains the decrease. The fourth and last stage is characterized by a zero $\xi$ value revealing that stationary stage is reached.

In contrast to what one can observe in the $\mathrm{Fe}-\mathrm{Cu}$ formation process, the tribochemical activity for the Fe-N system shown in Fig. 8 reveals large reactivity from the beginning of the process $\left(\frac{d \xi}{d t}\right.$ is large when $\left.t=0\right)$. Moreover, the reactivity seems to be proportional to the concentration gradient between the two phases $\left(\mathrm{Fe}\right.$ and $\left.\mathrm{Fe}_{2} \mathrm{~N}\right)$ and the reaction rate increases due to an ever growing interface between $\mathrm{Fe}_{2} \mathrm{~N}$ and $\mathrm{Fe}$ (grain size reduction). In fact, the $\mathrm{Fe}-\mathrm{Cu}$ is an endothermic system and energy storage is necessary in order to trigger the reaction. This does not occur in the exothermic $2 \mathrm{Fe}+\mathrm{Fe}_{2} \mathrm{~N}$ re- 
action. It can also be observed that the absolute value of $\xi_{F P}^{P}$ is a monotonic time function showing that saturation has not been achieved, and that there was no reduction of reagents reactivity capacity. The reaction stops because of the reactants extinction. This is another contrast to the Fe-Cu system in which a stationary stage is reached.

The presence of a stationary stage with zero $\xi$ in the end of the Fe$\mathrm{Cu}$ processing suggests us that there might be some unreacted amount of material left over. This fact calls for adjustment in eq. 2. Since the Fe-Cu reactions reached saturation, it is reasonable to believe that there should be a constant term in eq. 2 to account for the phase volume not transformed. Thus, the modified equation takes, the form:

$$
V(t)=k e^{-b t^{c}}+R
$$

and plugging this expression into eq. 18, we obtain:

$$
\xi_{\text {endo }}^{\varepsilon}(t) \propto-\frac{c b t^{c-1}}{1-k e^{-b t^{c}}-R\left[2-k e^{b t^{c}}(1-R)\right]} .
$$

On the other hand, the Fe-N system never reached saturation indicating that there shouldn't be any unreacted material left over and therefore $R$ should be zero for exothermic reactions reducing eq. 21 to the original one (eq. 2) and eq. 22 to:

$$
\xi_{e x o}^{\varepsilon}(t) \propto-\frac{c b t^{c-1}}{1-k e^{-b t^{c}}} .
$$

It is useful to analyze the limiting behavior of eqs. 22 and 23, For large $t$, eq. 22 becomes:

$$
\xi_{\text {endo }} \propto-\frac{t^{c-1}}{\text { const. }+\left(R-R^{2}\right) e^{t^{c}}} .
$$

For endothermic processes, $R>0$ and $|\xi| \sim 1 / e^{t^{c}} \rightarrow 0$, as expected. In eq. 23, the numerator dominates and $|\xi| \sim t^{c-1} \rightarrow \infty$, which is consistent with exothermic reactions. For small values of $t$, the denominator in eq. 22 and 23 is constant and then the numerator is dominant. The time derivative of $\xi$ when $t$ is small goes like $-t^{c-2}$. Therefore, for $c>2, d \xi / d t$ is zero in the beginning of the reaction, which is congruent with an energy storage stage which should be present in endothermic processes. On the other hand, for $c<2,|d \xi / d t|$ is maximum when $t \rightarrow 0$ which is compatible with exothermic reactions. 
To test these limits, fittings of the tribochemical activity time evolution curves in Figs. 5] and 8] to eqs. 22 and 23, respectively, were performed. It is important to point out that eqs. 22 and 23 are result of an empirical assumption and there is no argument to sustain that the fittings made here are, by any means, the best ones. One could obtain fittings as good as these ones using many different expressions. However, although the aim of the analysis made here is not to establish the absolute validity of the law in eqs. 2] and 21, it provides further insights on the usefulness of the tribochemical activity approach.

Table 2 shows the values of coefficients $k, b, c$, and $R$ obtained from numerical fitting of tribochemical activity time evolution curves to eqs. 22 and 23 where the coefficient $R$ applies to the former but not to the latter. Comparing the values obtained from the two Fe-Cu experiments (with 4 and 12 balls) we see that the values of coefficient $b$ are quite different, which is in accordance to the proposed relation of them to "work conditions". The values of $c$ from both experiments are very similar. This was expected since $c$ should be connected to impact energies and both experiments were performed with rotational frequency of $350 \mathrm{rpm}$. As a consequence, approximately the same amount of energy is transferred to the powder in both experiments and the local kinetics of both reactions are the same, despite the different number of balls. This analysis strengthens the suggestion that the shock frequency is only a milling condition and does not play a role in the energy transfer process, i.e., it is not a fundamental parameter of the process. The values of $k$ and $R$ are virtually the same since one process differs from the other by only the time scale. Moreover, according to tab. 2, $c$ is less than 2 for the

exothermic Fe-N system in contrast to the endothermic Fe-Cu system where $c$ is greater than 2 . These numbers are in accordance to the previous discussion. In this way, not only is $c$ connected to impact energies as suggested in [12, but also reflects the endothermic or exothermic nature of the reaction.

\section{Conclusions}

The formulation of the tribochemical activity concept provides a clear way to understand the evolution of phase transformations during MA. This is an initial attempt to describe the growth laws from global kinetics. Using this description it is possible to follow grain sizes, gradient of concentration and milling condition without chemical conditions. 
The time evolution of tribochemical activity allows the identification of four distinct stages in the milling of $\mathrm{Fe}-\mathrm{Cu}$ system: energy storage, reaction initiation and rate increase, reaction rate decrease, and stationary. These stages are in perfect agreement to the experimental observations. The energy storage stage is not identified in the $2 \mathrm{Fe}+\mathrm{Fe}_{2} \mathrm{~N} \longrightarrow \mathrm{Fe}_{4} \mathrm{~N}$ reaction because of the exothermic nature of this system. Moreover, the monotonous character of the $\xi(t)$ function shows that saturation is not achieved.

The striking difference between $\xi$ behavior for Fe- $\mathrm{N}$ and $\mathrm{Fe}-\mathrm{Cu}$ reflects the respective exothermic and endothermic nature of the reactions. Achievement of a saturation stage in $\mathrm{Fe}-\mathrm{Cu}$ processes suggests the existence of some amount of unreacted material left over. This fact calls for modification on the global kinetics law proposed in [12]. The small adjustment introduced extends the validity of the law to endothermic and exothermic processes and imposes a threshold to the characteristic exponent.

Further analysis of the two $\mathrm{Fe}-\mathrm{Cu}$ tribochemical activity curves suggests that impact frequency does not play an important role in the final product process. This is stressed by the values obtained from the numerical fitting of the phase volume and $\xi$ time evolutions.

At this point, it is really hard, and certainly premature, to assert that the tribochemical activity approach is definitive, but it definitely makes it clearer that the right path to fully understand the MA processes should bend more and more toward its description in terms of growth laws. Such description provides some clues about which parameters actually drive MA mechanisms, and also provides a means to separate out surface, mechanical impacts, and thermodynamics terms. The rather different tribochemical activity behav-

ior for the $\mathrm{Fe}-\mathrm{Cu}$ and $\mathrm{Fe}-\mathrm{N}$ systems might explain the broad diversity and incompatibility among various MA models present in the literature.

\section{References}

[1] Vasconcelos, I. F.; de Figueiredo, R. S. NanoStruc. Mat. 1999, 11, 935.

[2] Foct, J.; de Figueiredo, R. S. NanoStruc. Mat. 1994, 4, 685.

[3] Cocco, G.; Delogu, F.; Schiffini, L. J. Mater. Synth. Proces. 2000, 8, 167. 
[4] Silva, C. C.; Thomazini, D.; Pinheiro, A. G.; Lanciotti, F.; Sasaki, J. M.; Goes. J. C.; Sombra, A. S. B. J. Phys. Chem. Solids 2002, 63, 1745.

[5] Suryanarayana, C. Prog. Mater. Sci. 2001, 46, 1-184.

[6] Navarro, E.; Yavari, A. R.; Hernando, A. NanoStruc. Mat. 1999, 11, 81.

[7] Abdellaoui, M.; Gaffet, E. J. All. Comp. 1994, 209, 351.

[8] Abdellaoui, M.; Gaffet, E. Acta Metall. Mat. 1995, 43, 1087.

[9] Chen, Y.; Bibole, M.; Le Hazif, R.; Martin, G. Phys. Rev. B 1993 48, 14.

[10] Yavari, A. R.; Desré, P. J.; Benameur, T. Phys. Rev. Lett. 1992, 68, 2235.

[11] Yavari, A. R. Mat. Sci. Eng. 1994, A179, 20.

[12] Matteazzi, P.; Basset, D.; Miani, F.; Le Caër, G. NanoStruc. Mat. 1993 2,213 .

[13] Girot, T.; Begin-Colin, S.; Devaux, J.; Le Caër, G.; Mocellin, A. J. Mat. Synt. Proc. 2000, 8, 139.

[14] Takacs, L. Prog. Mat. Sci. 2002, 47, 355.

[15] Delogu, G.; Cocco, G. J. Mater. Synth. Proces. 2000, 8, 271.

[16] Le Caër, G. Private communication

[17] Jiang, J. Z.; Gonser, U.; Gente, C.; Bormann, R. Appl. Phys. Lett. 1993, 63, 1056.

[18] Foct, J.; de Figueiredo, R. S.; Richard, O.; Morniroli, J. P. Proceedings of 1st International Conference on Mechanochemistry (INCOME) 1993, 2,42 , Slovakia.

[19] de Figueiredo, R. S.; Foct, J. International Symposium on Metasteble, Mechanically Alloyed and Nanocrystalline Materials (ISMANAN94) 1994, France. 
[20] de Figueiredo, R. S.; Foct, J. Materials Science Forum 1995, 179-181, 219.

[21] Foct, J.; de Figueiredo, R. S.; 4th International Conference on High Nitrogen Steels HNS 95 1995, Kyoto, Japan.

[22] Foct, J.; de Figueiredo, R. S.; ISIJ International 1996, 36, 962.

[23] Kano, M; J. Alloys Comp. 2001, 327, 43.

[24] de Figueiredo, R. S. Mécanosynthèse d'Alliages Binaires et Ternaires FeMe- $N$ Charactérisée et Discutée à partir des Résultats de Spectrométrie Mössabuer, PhD Dissertation, 1995, N1540, USTL France.

[25] Huang, J. Y.; He, A. Q.; Wu, Y. K.; Ye, H. Q.; Li, D. X. J. Mat. Sci 1996, 31, 4165 .

[26] Uenishi, K.; Kobayashi, K. F.; Nasu, S.; Hatano, H.;Ishihara, K. N.; Shingu, P. H. Z. Metallkd. 1992, 83, 132. 
Table 1: Grain size for some Fe-Cu milled samples with 12 balls and 350 $\mathrm{rpm}$. This results were obtained refining the x-rays patterns using Rietveld method.

\begin{tabular}{ccc}
\hline \multirow{2}{*}{ time $(\mathbf{h})$} & \multicolumn{2}{c}{ grain size (nm) } \\
\cline { 2 - 3 } & bcc phase & fcc phase \\
\hline 1 & 74 & 44 \\
2 & 41 & 38 \\
4 & 33 & 24 \\
8 & 15 & 14 \\
12 & 14 & 14 \\
16 & - & 13 \\
\hline
\end{tabular}

Table 2: Coefficients obtained by fitting Figs. $[5$ and 8 to eqs. 22 and 23 , respectively.

\begin{tabular}{ccccc}
\hline & $\mathbf{k}$ & $\mathbf{b}$ & $\mathbf{c}$ & $\mathbf{R}$ \\
\hline $\mathrm{Fe}-\mathrm{Cu}(4 \mathrm{~b})$ & 0.479 & 0.003 & 2.729 & 0.075 \\
$\mathrm{Fe}-\mathrm{Cu}(12 \mathrm{~b})$ & 0.485 & 0.121 & 2.658 & 0.069 \\
$\mathrm{Fe}-\mathrm{N}$ & 0.497 & 0.106 & 1.661 & - \\
\hline
\end{tabular}




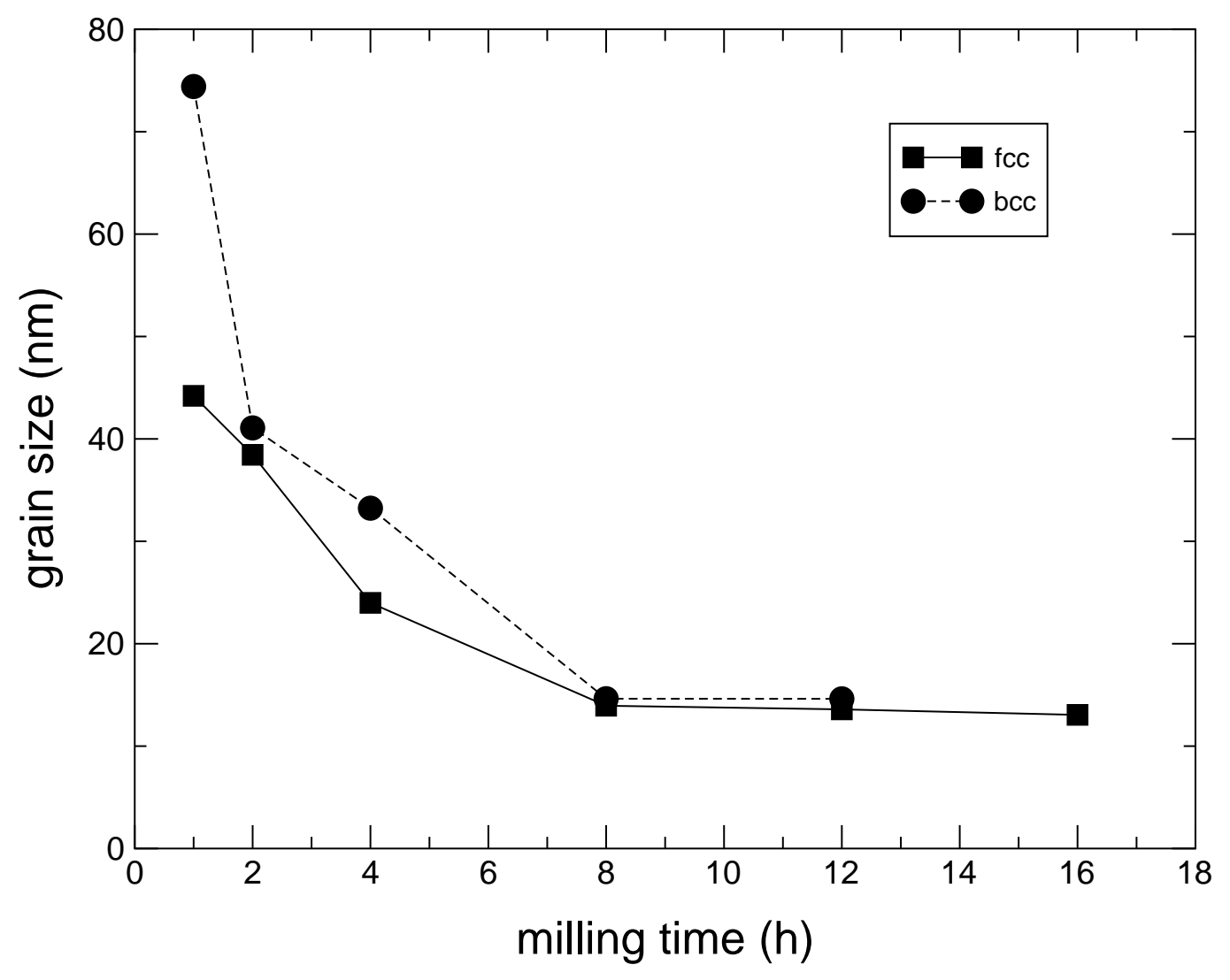

Figure 1: Grain size evolution of bcc and fcc phases during milling for Fe$\mathrm{Cu}$ milled with $350 \mathrm{rpm}$ and 4 balls revealing a similar behavior for the two phases. 


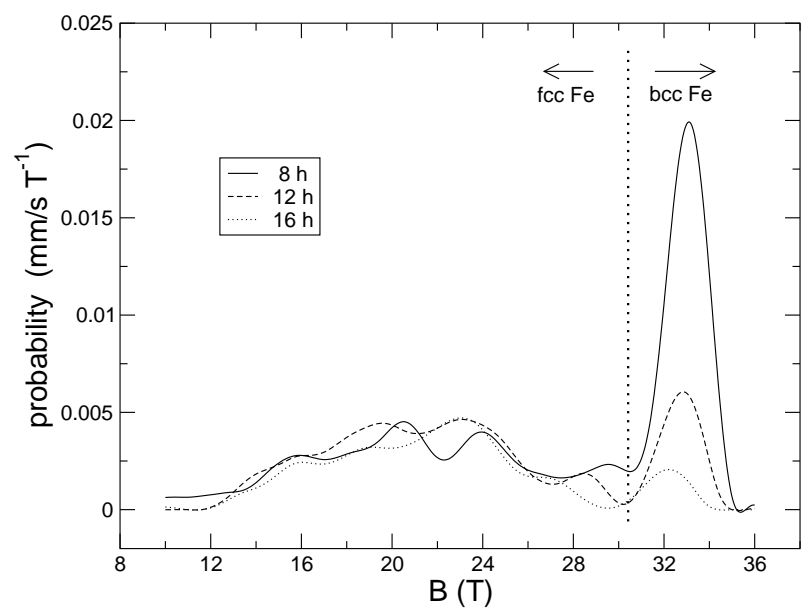

(a) $350 \mathrm{rpm}-4$ balls

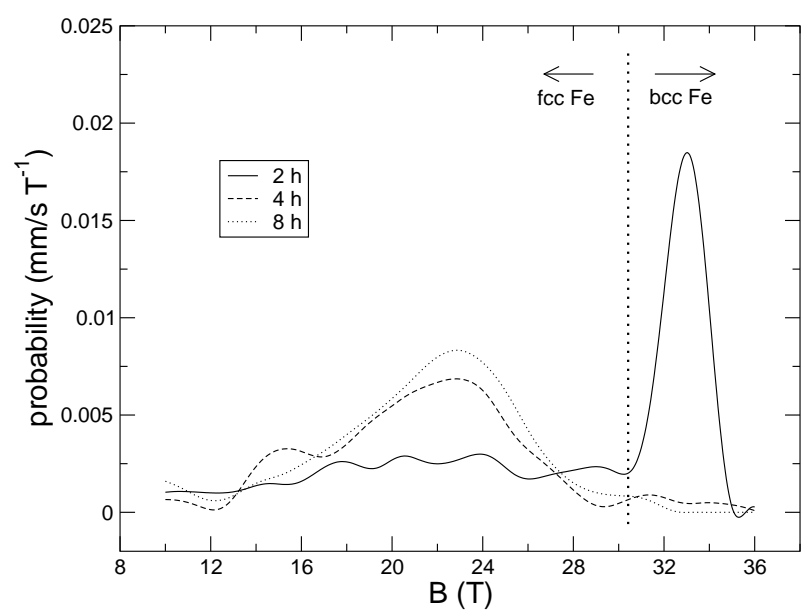

(b) 350 rpm - 12 balls

Figure 2: BHF distributions for some Fe-Cu milled samples showing a clear separation between Fe-fcc and Fe-bcc phases (low and high BHF values respectively) 


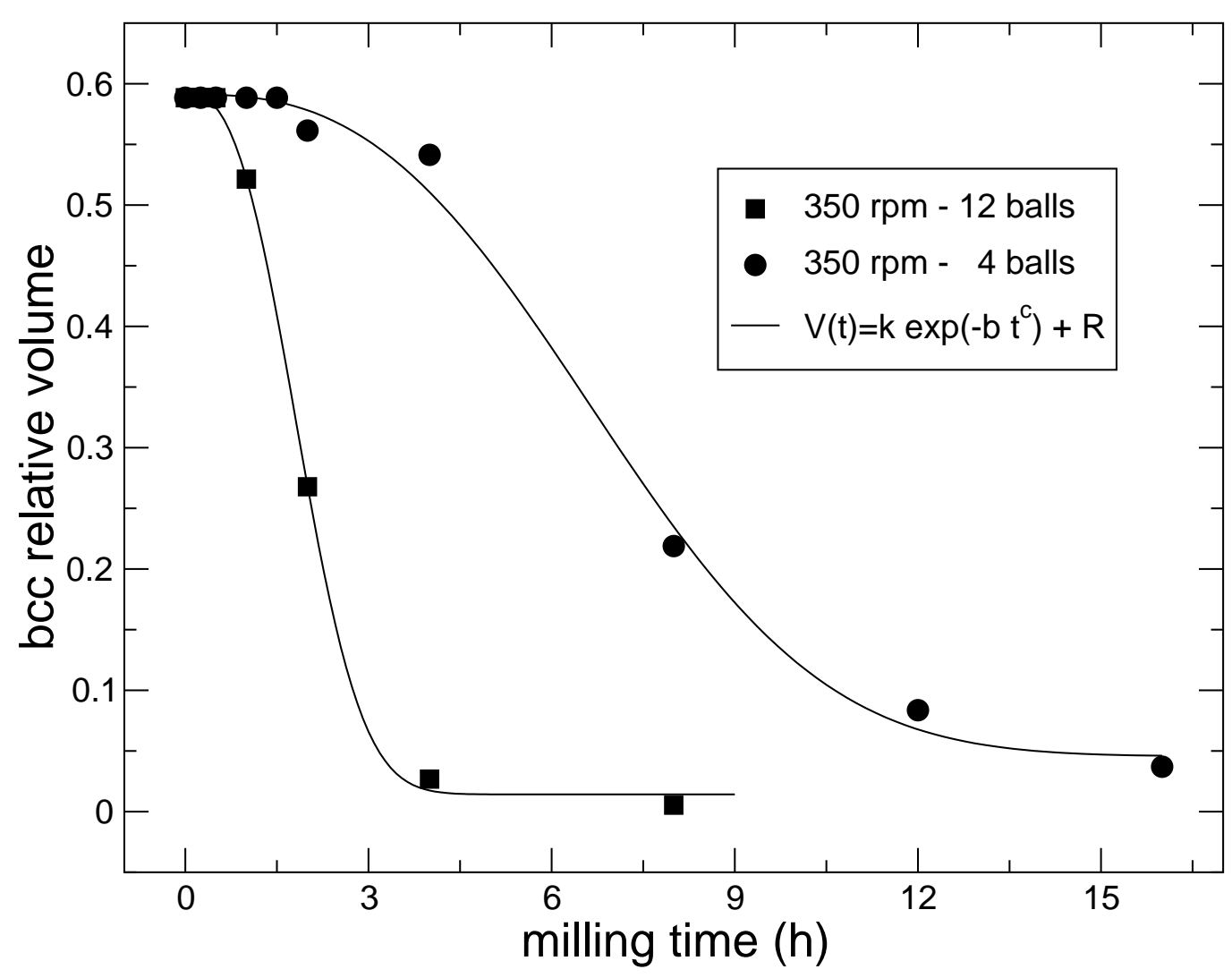

Figure 3: Bcc relative volume phase for the $\mathrm{Fe}-\mathrm{Cu}$ system obtained by Mössbauer spectroscopy. 


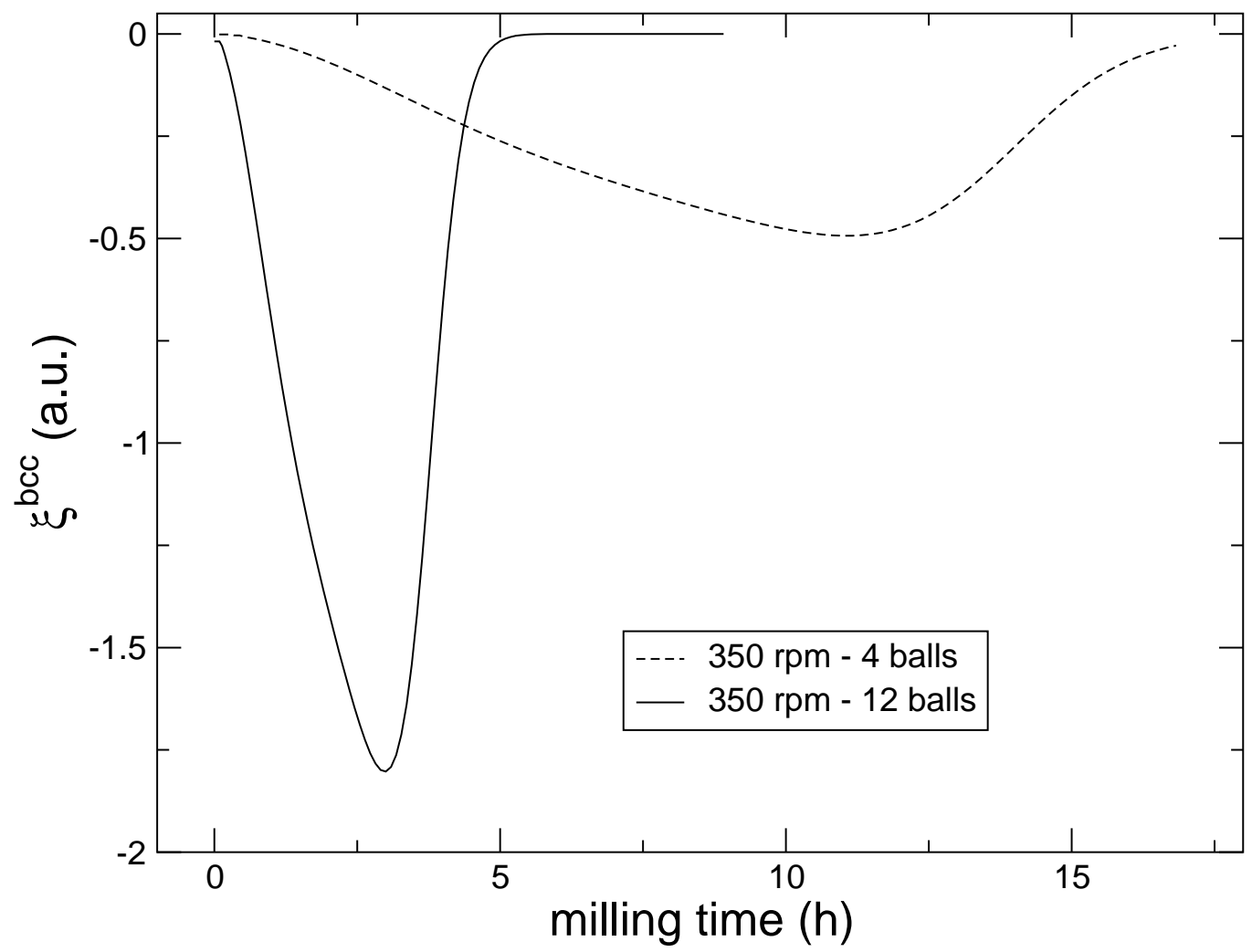

Figure 4: Tribochemical activity to destroy the bcc phase in the Fe-Cu system as a function of milling time. The curves were calculated from data in Fig. 3 using eq. 19 . 


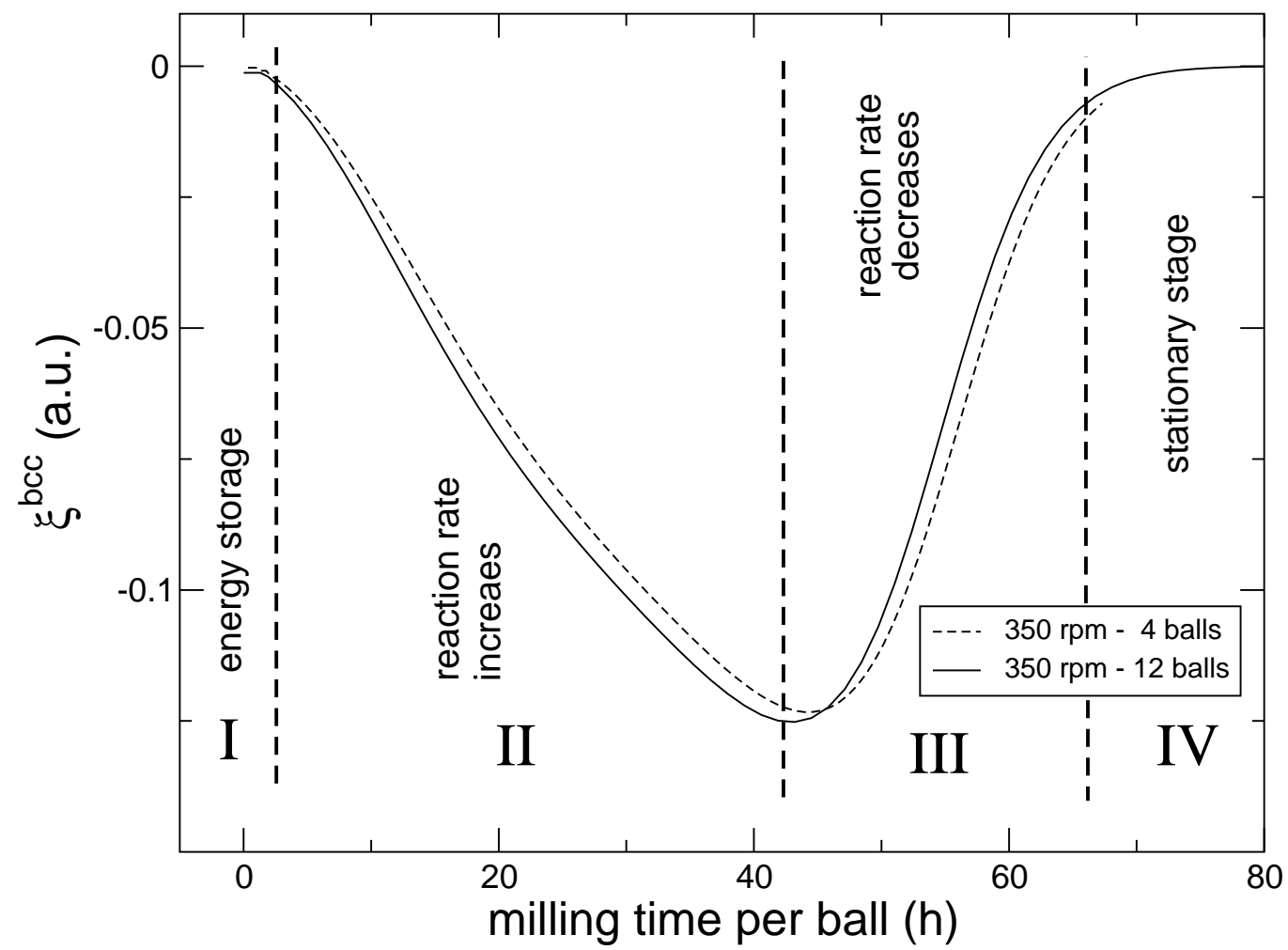

Figure 5: Tribochemical activity to destroy the bcc phase in the Fe-Cu system as a function of milling time rescaled by the number of balls. 


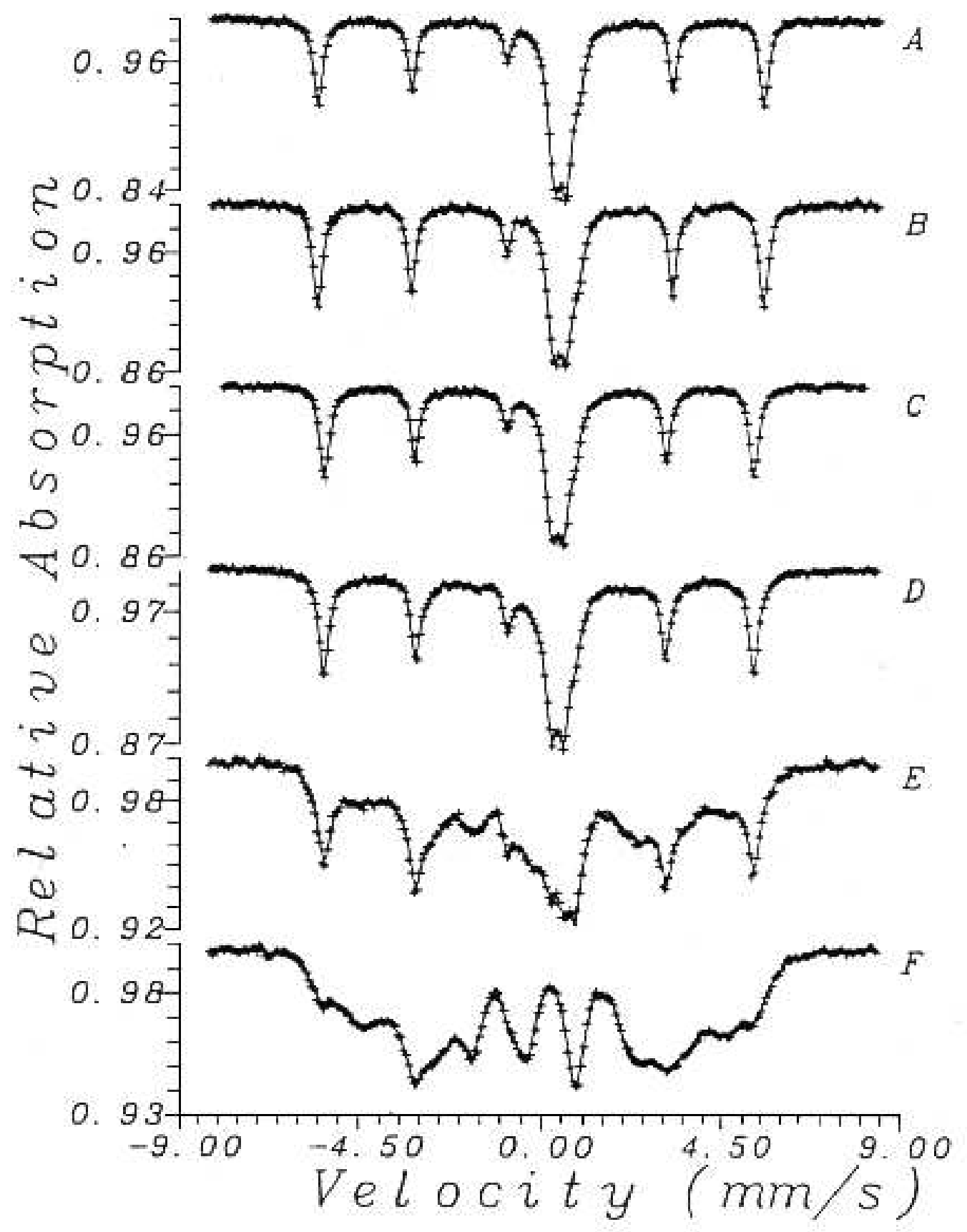

Figure 6: Mössbauer spectra at room temperature for milled $\mathrm{Fe}_{2} \mathrm{~N}+2 \mathrm{Fe}$ up to (a) $5 \mathrm{~min}$; (b) $20 \mathrm{~min}$; (c) $1 \mathrm{~h}$; (d) $2 \mathrm{~h}$; (e) $4 \mathrm{~h}$ and (f) $16 \mathrm{~h}$ 


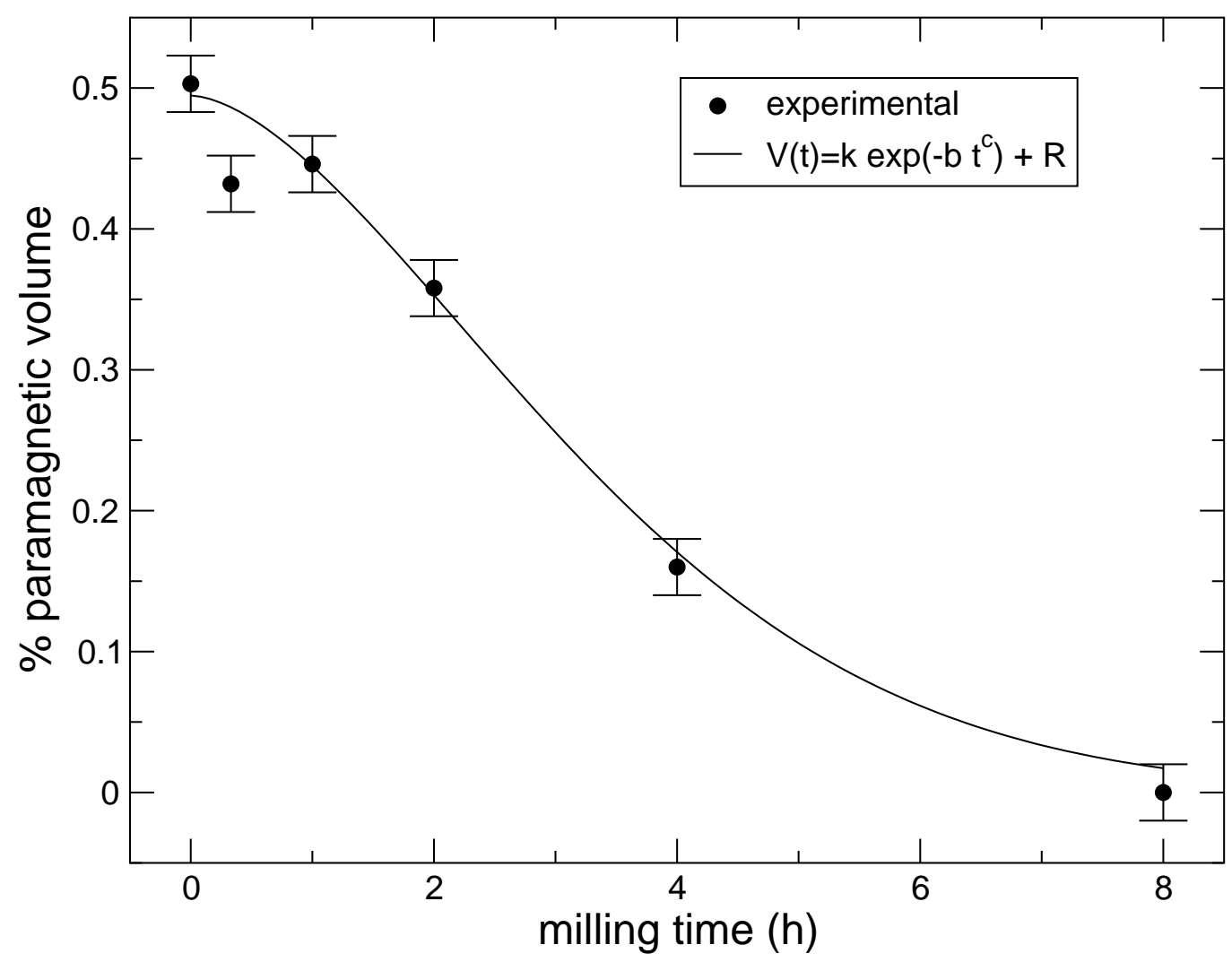

Figure 7: Paramagnetic relative volume phase for the Fe-N system obtained by Mössbauer spectroscopy 


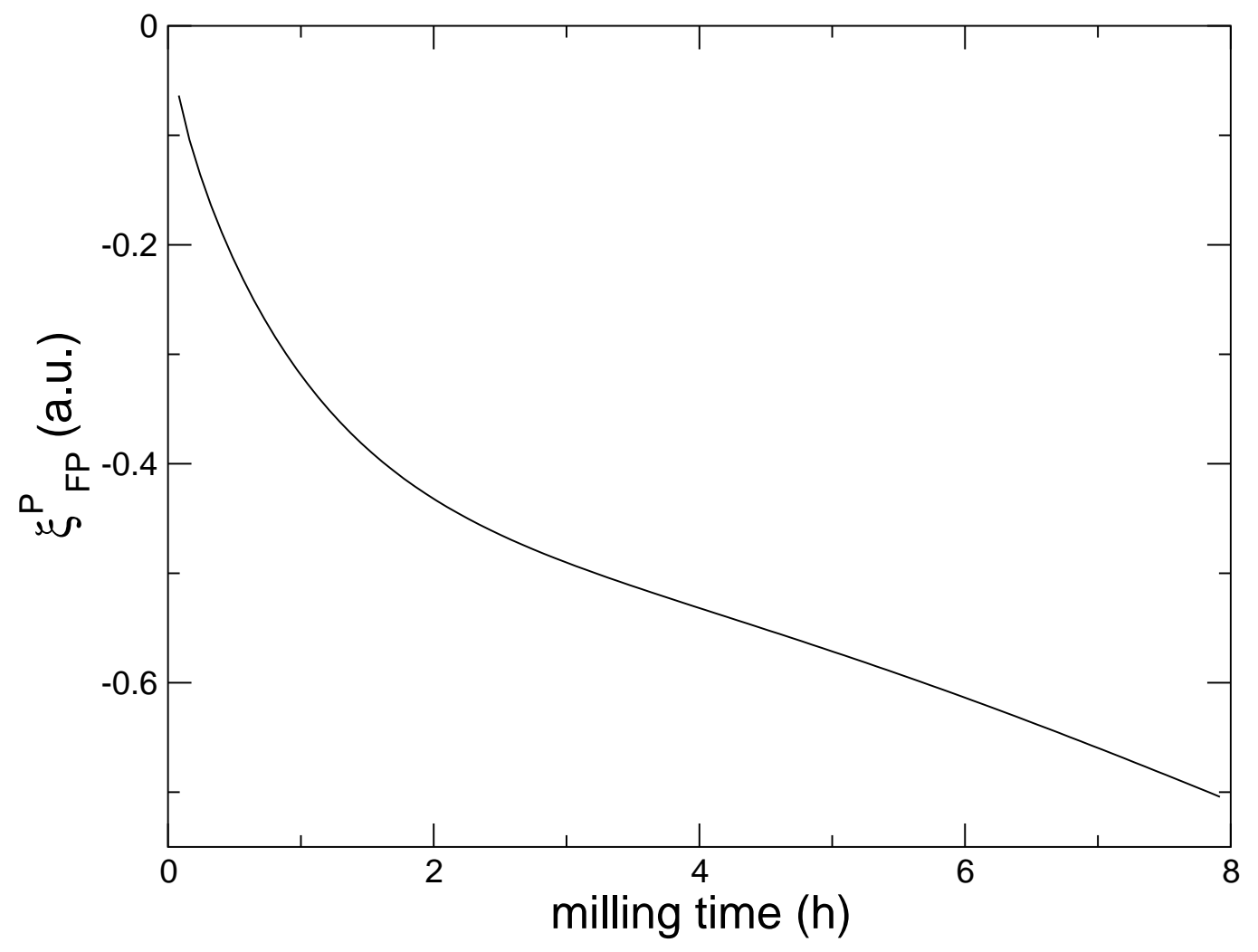

Figure 8: Time evolution of tribochemical activity to destroy the paramagnetic phase in the Fe-N system. The curve was calculated from data in Fig. 7 using eq. 20. 